\title{
ARITHMETIC AND ANALYTIC PROPERTIES OF PAPER FOLDING SEQUENCES
}

\section{Mendes France and A.J. van der Poorten}

\author{
To Kurt Mahler
}

\begin{abstract}
The mechanical procedure of paper folding generates an uncountable family of infinite sequences of fold patterns. We obtain the associated Fourier series and show that the sequences are almost periodic and hence deterministic. Further, we show that paper folding numbers defined by the sequences are all transcendental.
\end{abstract}

\section{Introduction}

In his first paper, more than fifty years ago, Mahler [7] computed the Wiener spectrum of a certain sequence (see [12]). A little later, Mahler [8] introduced a method for proving the transcendence of values of a class of related functions (see the survey [6], Chapter 15).

We study a family of sequences generated by a mechanical procedure paper folding. We obtain the Fourier series for each paper folding sequence

$$
f=\left(f_{n}\right) \in\{-1,+1\}^{N}
$$

and find that the sequences are almost-periodic (in the sense of Besicovitch). For each algebraic $\alpha, 0<|\alpha|<1$ we show that the number

Received 6 March 1981. 


$$
\sum_{n=1}^{\infty} f_{n} \alpha^{n}
$$

is transcendental. Since, moreover, we see that there is a natural one-toone correspondence between numbers in the interval $[0,1]$ and paper folding sequences this yields a map by each $\alpha$, as above, of $[0,1]$ into the transcendental numbers.

\section{Paper folding sequences ([2], [3], [9], [10])}

The folding operator $F$ acting on a sheet of paper $P$ can fold either positively or negatively:
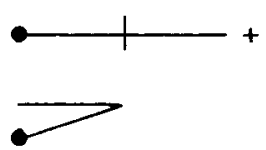
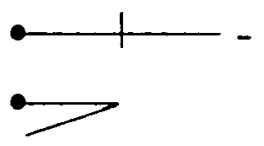

We denote the $n$th fold by $F_{\varepsilon(n)}$ where $\varepsilon(n)=+1$ if the fold is positive, and $\varepsilon(n)=-1$ if it is negative. When we have performed the operations $F_{E(1)}, \ldots, F_{E(N)}$ on $P$, the paper

$$
P\left(\prod_{n=1}^{N} F_{\varepsilon(n)}\right)
$$

when unfolded shows a sequence of $2^{N}-1$ ridges $r$ and valeys $v$. If, as usual, we deem two sequences on the alphabet $\{r, v\}$ to be "close together" if they commence with the same word (see, for example [10]), thẹ we readily see that for any infinite sequence $\varepsilon=\left(\varepsilon_{n}\right) \in[-1,+1\}^{N}$ the set

$$
\left\{P\left(\prod_{n=1}^{N} F_{\varepsilon(n)}\right): N=1,2,3, \ldots\right\}
$$

has cluster points. Any such cluster point

$$
f=f_{1} f_{2} f_{3} \ldots \in\{r, v\}^{N}
$$

is called a paper folding sequence. In the sequel we write $v=+1$, $r=-1$. 
A paper folding sequence is characterised by the property that it may be "unfolded" arbitrarily many times, yet remain a paper folding sequence. Formally, we introduce the operator $T$ defined on $\{-1,+1\}^{N}$ by

$$
T:\left(g_{n}\right) \rightarrow\left(g_{2 n}\right) \text {. }
$$

Then $f=\left(f_{n}\right)$ is a paper folding sequence if and only if

(i) $f_{2 n+1}=(-1)^{n} f_{1} \quad(n \geq 0)$,

(ii) Tf is a paper folding sequence.

It follows that a paper folding sequence is determined by its subsequence $f_{1} f_{2} f_{4} \cdots f_{2} \ldots$. Thus, given a binary "decimal" $0 . a_{0} a_{1} a_{2} \cdots$, $a_{i} \in\{0,1\}$ we have a correspondence with a paper folding sequence $\left(f_{n}\right)$ by way of $f_{2^{k}}=2 a_{k}-1 \quad(k \geq 0)$ and $f_{2^{k}(2 l+1)}=(-1)^{z_{f}}{ }_{2^{k}}(k \geq 0)$.

The following result is an easy consequence of the above characterisation.

LEMMA. A paper folding sequence is not eventually periodic.

Proof. Suppose to the contrary that $2^{a}(2 b+1)$ be a period. Then (writing $f(n)$ in place of $f_{n}$ ),

$$
f\left(n+2^{a}(2 b+1)\right)=f(n) \text { for all } n>n_{0}
$$

In particular, choosing $n=2^{a+1} m$ we have

$$
f\left(2^{a}(2 m+2 b+1)\right)=f\left(2^{a+1} m\right), m>m_{0}
$$

But the sequence $T^{a} f$ defined by $n \rightarrow f\left(2^{a} n\right)=T^{a} f(n)$ is again a paper folding sequence, so

$$
T^{a} f(2 m+2 b+1)=(-1)^{m+b} T^{a} f(1)=T^{a+1} f(m), \quad m>m_{0} .
$$

Hence the paper folding sequence $T^{a+1} f$ is an alternating sequence (which forces $T^{a+2} f$ to be constant) and this is a contradiction. 


\section{Fourier series of paper folding sequences}

We recall that the Fourier-Bohr coefficient $\hat{f}(x)$ of $f$ at the point $x$, for $x \in \Pi=R / \mathbb{Z}$, is given by the limit (if it exists):

$$
\lim _{N \rightarrow \infty} \frac{1}{N} \sum_{n \leq N} f(n) \exp (-2 i \pi n x)
$$

We compute

$$
\begin{aligned}
R_{N}^{f}(x) & =\sum_{n \leq N} f(n) \exp (-2 i \pi n x) \\
& =\sum_{n \leq N / 2} f(2 n) \exp (-2 i \pi \cdot 2 n x)+\sum_{n \leq N / 2} f(2 n+1) \exp (-2 i \pi \cdot(2 n+1) x)+o(1) \\
& =R_{N / 2}^{T f}(2 x)+f(1) e^{-2 i \pi x} \sum_{n \leq N / 2}(-1)^{n} e^{-4 i \pi n x}+o(1) \\
& =R_{N / 2}^{T f}(2 x)+f(1) e^{-2 i \pi x} \sum_{n \leq N / 2} \exp 2 i \pi\left(\frac{1}{2}-2 x\right) n+o(1) .
\end{aligned}
$$

The sum is $N / 2+O(1)$ or $O(1)$ according as $\frac{2}{2}-2 x \in \mathbb{Z}$ or not. We write

$$
X(x)= \begin{cases}1 & \text { if } 2 x \in \frac{1}{2}+\mathbb{Z}, \\ 0 & \text { otherwise, }\end{cases}
$$

and obtain

$$
R_{N}^{f}(x)=R_{N / 2}^{T f}(2 x)+f(1) e^{-2 i \pi x} \frac{N}{2} \chi(x)+O(1)
$$

Iterating this relation yields

$$
R_{N}^{f}(x)=\left(\sum_{i=0}^{k-1} f\left(2^{l}\right) e^{-2 i \pi x 2^{2}} \frac{1}{2^{l+1}} \cdot x\left(x 2^{z}\right)\right) N+R_{N / 2^{k}}^{T^{k}} f^{k}\left(x 2^{k}\right)+o(k)
$$

Taking $k=[\log N]$ and dividing by $N$, we have

$$
\hat{f}(x)=\lim _{N \rightarrow \infty} \frac{1}{N} R_{N}^{f}(x)=\sum_{l=0}^{\infty} \frac{f\left(2^{2}\right)}{2^{l+1}} e^{-2 i \pi x 2^{2}} \times\left(x 2^{l}\right) .
$$

But 


$$
\begin{aligned}
x\left(x 2^{2}\right)=1 & \Leftrightarrow 2 x \cdot 2^{z} \in \frac{1}{2}+\mathbb{Z} \\
& \Leftrightarrow x=\frac{2 a+1}{2^{z+2}}, \quad a \in \mathbb{Z} .
\end{aligned}
$$

Hence

$$
\hat{f}(x)= \begin{cases}-i(-1)^{a} \frac{f\left(2^{\nu}\right)}{2^{\nu+1}} & \text { if } x=\frac{2 a+1}{2^{\nu+2}}, v \geq 0, \\ 0 & \text { otherwise. }\end{cases}
$$

Thus the Fourier spectrum of $f$,

$$
S=\operatorname{sp}(f)=\{x: \hat{f}(x) \neq 0\},
$$

consist of the dyadic points in $\pi$ with denominator greater than or equal to 4 .

We observe that

$$
\begin{aligned}
\sum_{x \in X}|\hat{f}(x)|^{2} & =\sum_{\nu=0}^{\infty} \sum_{a=0}^{2^{\nu+1}}\left(\frac{1}{2^{\nu+1}}\right)^{2}=\sum_{\nu=0}^{\infty} \frac{2^{\nu+1}}{\left(2^{v+1}\right)^{2}} \\
& =\sum_{\nu=0}^{\infty} \frac{1}{2^{\nu+1}}=1=\|f\|^{2},
\end{aligned}
$$

the norm \|\| being defined as usual by

$$
\|f\|=\lim _{N \rightarrow \infty} \sup \left(\frac{1}{N} \sum_{n \leq N}|f(n)|^{2}\right)^{\frac{2}{2}} .
$$

It follows that $f$ is Besicovitch almost periodic:

$$
\begin{aligned}
f(n) & =\sum_{x \in S} \hat{f}(x) e^{2 i \pi n x} \\
& =i \sum_{\nu=0}^{\infty} 2^{\nu+1} \sum_{a=0}^{-1}(-1)^{a} \frac{f\left(2^{\nu}\right)}{2^{\nu+1}} \exp \left(2 i \pi \frac{2 a+1}{2^{\nu+2}} n\right),
\end{aligned}
$$

the implied convergence being \|\| convergence. However, in

$$
-i \sum_{\nu=0}^{\infty} f\left(2^{\nu}\right) \exp \left(2 i \pi \frac{n}{2^{\nu+2}}\right) \cdot\left(\frac{1}{2^{v+1}} \sum_{a=0}^{2^{\nu+1}-1}(-1)^{a} \exp \left(2 i \pi \frac{a n}{2^{\nu+1}}\right)\right) \text {, }
$$

the inner sum $A(\nu, n)$ is 


$$
A(\nu, n)=\frac{1}{2^{\nu+1}} \sum_{a=0}^{2^{\nu+1}-1} \exp \left(\frac{2 i \pi}{2^{\nu+1}}\left(2^{\nu}+n\right) a\right)
$$

which is 1 if $2^{\nu+1}$ divides $2^{\nu}+n$ and 0 otherwise. Hence

$$
\begin{aligned}
A(\nu, n)=1 & \Leftrightarrow \frac{n}{2^{v+1}} \epsilon \frac{1}{2}+\mathbb{Z} \\
& \Leftrightarrow \nu=v_{2}(n),
\end{aligned}
$$

where $\nu_{2}(n)$ is the 2-valuation of $n$ (meaning that $2^{\nu_{2}(n)}$ "exactly divides" $n$ ). Thus the rearranged Fourier series sums to

$$
-i f\left(2^{\nu_{2}(n)}\right) \exp 2 i \pi \frac{n}{2^{\nu_{2}(n)+2}}=f\left(2^{\nu_{2}(n)}\right) i\left(n / 2^{\nu_{2}(n)}\right)-1 \text {, }
$$

a quantity easily seen to equal $f(n)$ in view of the characterisation of paper folding sequences given above. This is to say that after our rearrangement we actually obtain pointwise convergence of the Fourier series.

We have shown:

THEOREM 1. Let $f=(f(1), f(2), \ldots)$ be a paper folding sequence. Then $f$ is almost periodic (in the sense of Besicovitch)

$$
f(n)=\sum_{x \in S} \hat{f}(x) \exp (2 i \pi n x)
$$

where $S$ is the set of dyadic rationals in $\pi$ with denominator greater than or equal to 4 . Explicitly,

$$
\hat{f}(x)= \begin{cases}-i(-1)^{a} f\left(2^{\nu}\right) / 2^{\nu+1} & \text { if } x=\frac{2 a+1}{a^{\nu+2}}, \nu \geq 0, \\ 0 & \text { in alz other cases. }\end{cases}
$$

This result provides an indirect proof of the lemma of Section 2.

COROLLARY 1. $f$ is not ultimately periodic.

We may also read off the nature of the (Wiener) spectral measure $\Lambda$ belonging to a paper folding sequence:

COROLLARY 2. The spectral measure $\Lambda$ "lives" on $S$ and 
$\Lambda\left(\left\{\frac{2 a+1}{2^{v}}\right\}\right)=\frac{1}{2^{2 v-2}}$

Moreover, Besicovitch almost periodic sequences are deterministic (in the sense of Kamae, Rauzy and Weiss (see [4] and [11])):

COROLLARY 3. Paper folding sequences are deterministic.

\section{Transcendence of paper folding numbers}

Given a paper folding sequence $f=f^{(0)}$ we obtain a sequence $f^{(1)}=T f, f^{(2)}=T^{2} f, \ldots$ of paper folding sequences. To each sequence $f^{(k)}$ we associate its generating function

$$
F_{k}(x)=\sum_{n=1}^{\infty} f_{n}^{(k)} x^{n} \quad(k \geq 0),
$$

and we note that our characterisation of paper folding sequences is equivalent to the recursive system of functional equations:

$$
F_{k}\left(x^{2}\right)=F_{k-1}(X)-f_{1}^{(k-1)} X /\left(x^{2}+1\right) \quad(k \geq 1) .
$$

Such systems are studied in [5], which yields:

THEOREM 2. If $\left(f_{n}\right)$ is a paper folding sequence and $\alpha$ is algebraic with $0<|\alpha|<1$ then

$$
\sum_{n=1}^{\infty} f_{n} \alpha^{n}
$$

is transcendental.

$$
\text { Writing } G(X)=\frac{2}{2}(F(X)+1 /(1-X))=\sum_{n=1}^{\infty} g_{n} X^{n} \text { we obtain a paper }
$$

folding sequence $g=\left(g_{n}\right) \in\{0,1\}^{N}$, and the transcendence of all paper folding numbers

$$
0 \cdot g_{1} g_{2} g_{3} \ldots
$$

regardless of the base implied by the "decimal" point.

To prove the theorem given [5], we have only to show that the sequence 
$\left(F_{k}\right)$ is strongly transcendental ([5], p. 23). This is a technical condition requiring that the $F_{k}$ should not be arbitrarily well approximable by algebraic functions. Precisely, let $P_{j}(X ; F) \quad(0 \leq j \leq \rho)$ be polynomials in $X$ and in a given finite set of the coefficients of the series $F$, and write

$$
\sum_{j=0}^{\rho} P_{j}\left(X ; F_{k}\right)\left(F_{k}(X)\right)^{j}=\sum_{\mu=0}^{\infty} p_{\mu}(k) X^{\mu} .
$$

Then there should be a constant $m$ (depending only on the $P_{j}$ ) so that there is a $\mu$ with $\mu \leq m$ and $p_{\mu}(k) \neq 0$, whenever not all the $P_{j}\left(X ; F_{k}\right)$ are identically zero.

Firstly, since each coefficient of the $E_{k}$ takes one of only two possible values it suffices for us to restrict our attention to a subsequence $(k(n))$ on which the $P_{j}$ are independent of $k$ and are not all zero. Next, on similar grounds, it is plain that the sequence $\left(F_{k(n)}\right)$ has subsequences that converge to a paper folding function. (As usual, two formal power series are "close together" if they commence with the same terms.) Thus if the sequence $\left(F_{k}\right)$ is not strongly transcendental there is a paper folding function $F$ algebraic over the field of rational functions.

But a power series with integer coefficients and radius of convergence 1 is either a rational function or has the unit circle as its natural boundary (Carlson [1]). Thus $F$ must be rational, and so its coefficients (from a certain point on) satisfy a linear homogeneous recursion with constant coefficients. In the present case, where the coefficients of $F$ are +1 or -1 , the sequence has to be (eventually) periodic. This contradiction completes the proof of Theorem 2 .

\section{References}

[1] Fritz Carlson, "Über Potenzreihen mit ganzzahligen Koeffizienten", Math. 2. 9 (1921), 1-13. 
[2] Chandler Davis, Donald E. Knuth, "Number representations and dragon curves - I", J. Recreational Math. 3 (1970), 61-81; "Number representations and dragon curves - II", J. Recreational Math. 3 (1970), 133-149.

[3] F.M. Dekking, "Constructies voor 0-1-rijen met strikt ergodische afgesloten baan" (Doctoraalscriptie, 1974 ).

[4] Teturo Kamae, "Subsequences of normal sequences", Israel J. Math. 16 (1973), 121-149.

[5] J.H. Loxton and A.J. van der Poorten, "Arithmetic properties of certain functions in several variables III", BuZZ. Austral. Math. Soc. 16 (1977), 15-47.

[6] J.H. Loxton and A.J. van der Poorten, "Transcendence and algebraic independence by a method of Mahler", Transcendence theory: advances and applications, 211-226 (Proc. Conf. Univ. Cambridge, Cambridge, 1976. Academic Press, London, 1977).

[7] Kurt Mahler, "On the translation properties of a simple class of arithmetical functions", J. Math. Phys. 6 (1972), 158-163.

[8] Kurt Mahler, "Arithmetische Eigenschaften der Lösungen einer Klasse von Funktionalgleichungen", Math. Ann. 101 (1929), 342-366.

[9] M. Mendès-France, "Principe de la symétrie perturbée", Séminaire de Théorie des Nombres, Paris 1979-80, 77-98 (Séminaire DelangePisot-Poitou. Birkhäuser, Boston, Basel, Stuttgart, 1981).

[10] M. Mendès France et $G$. Tenenbaum, "Dimension des courbes planes, papiers pliés et suites de Rudin-Shapiro", Bulz. Soc. Math. France (to appear).

[11] G. Rauzy, "Nombres normaux et processus déterministes", Acta Arith. $29(1976), 211-225$.

[12] Norbert Wiener, "Generalized harmonic analysis", Acta Math. 55 (1930), $117-258$.

UER de Mathématiques et d'Informatique, Université de Bordeaux 1 , 351 Cours de la Libération, 33405 Talence - Cédex, France;
School of Mathematics and Physics, Macquarie University, North Ryde, New South Wales 2113, Australia. 